\title{
KULEUVEN
}

\section{Individual loss reserving using paid-incurred data}

Mathieu Pigeon, Katrien Antonio and Michel Denuit 


\title{
Individual loss reserving using paid-incurred data
}

\author{
Mathieu Pigeon ${ }^{1}$, Katrien Antonio ${ }^{2}$, and Michel Denuit ${ }^{1}$ \\ ${ }^{1}$ Institute of Statistics, Biostatistics and Actuarial Sciences (ISBA), Université \\ catholique de Louvain (UCL), Belgium \\ ${ }^{2}$ Faculty of Economics and Business, KU Leuven, Belgium, and Faculty of Economics \\ and Business, University of Amsterdam, The Netherlands
}

February 25, 2014

\begin{abstract}
In this paper, the individual claim reserving model proposed by Pigeon et al. (2013) is extended to include paid and incurred data. Analytic expressions are derived for the expected ultimate losses, given observed development patterns. The usefulness of this new model is illustrated using a portfolio of general liability insurance policies. Detailed comparisons with existing approaches reveal that the paid-incurred individual reserving method proposed in this paper performs well and produces more accurate predictions.
\end{abstract}

Keywords: Stochastic Loss Reserving, General Insurance, Multivariate Skew Normal distribution, Prediction.

\section{Introduction}

An insurance company is liable for the claims generated by the contracts sold to clients. The insurer will hold capital aside to meet future liabilities attached to incurred claims. He thus must predict, with maximum accuracy, the total amount necessary to pay claims that he has legally committed to cover for. This is the job of a reserving actuary. Our paper presents a novel framework for reserving using individual claim data, combining two sources of information, namely claim payments and incurred losses. On the one hand, we extend the framework developed in Pigeon et al. (2013) for reserving with individual claims, and now enable consistent handling of paid and incurred information. On the other hand, we extend remarkable strategies for claims reserving with paid-incurred information summarized in run-off triangles (see Quarg and Mack (2004), Wüthrich and Merz (2010), Posthuma et al. (2008)) to the setting of individual claims.

Figure 1 illustrates the run-off (or development) process of a non-life insurance claim. A claim occurs at a certain point in time $\left(t_{1}\right)$, consequently it is declared to the insurer (at $\left.t_{2}\right)$, possibly after a period of delay, and one or several claim payments follow (at time points $t_{3}, t_{4}, t_{5}$ ) until the settlement (i.e. closure, at time $t_{6}$ ) of the claim. Insurance companies distinguish Reported But Not Settled (RBNS) claims and Incurred But Not Reported (IBNR) claims. For an RBNS claim occurrence and declaration take place before the present moment (say $\tau$ ) and the 
settlement occurs afterwards (i.e. $\tau \geqslant t_{2}$ and $\tau<t_{6}$ in Figure 1). An IBNR claim has occurred before the present moment, but its declaration and settlement follow afterwards (i.e. $t_{1} \leq \tau<t_{2}$ in Figure 1). The majority of techniques for loss reserving (see Wüthrich and Merz (2008)) aggregate information on the development of individual claims into run-off triangles. Figure 1 visualizes this operation and Figure 2 is an example of a run-off triangle.

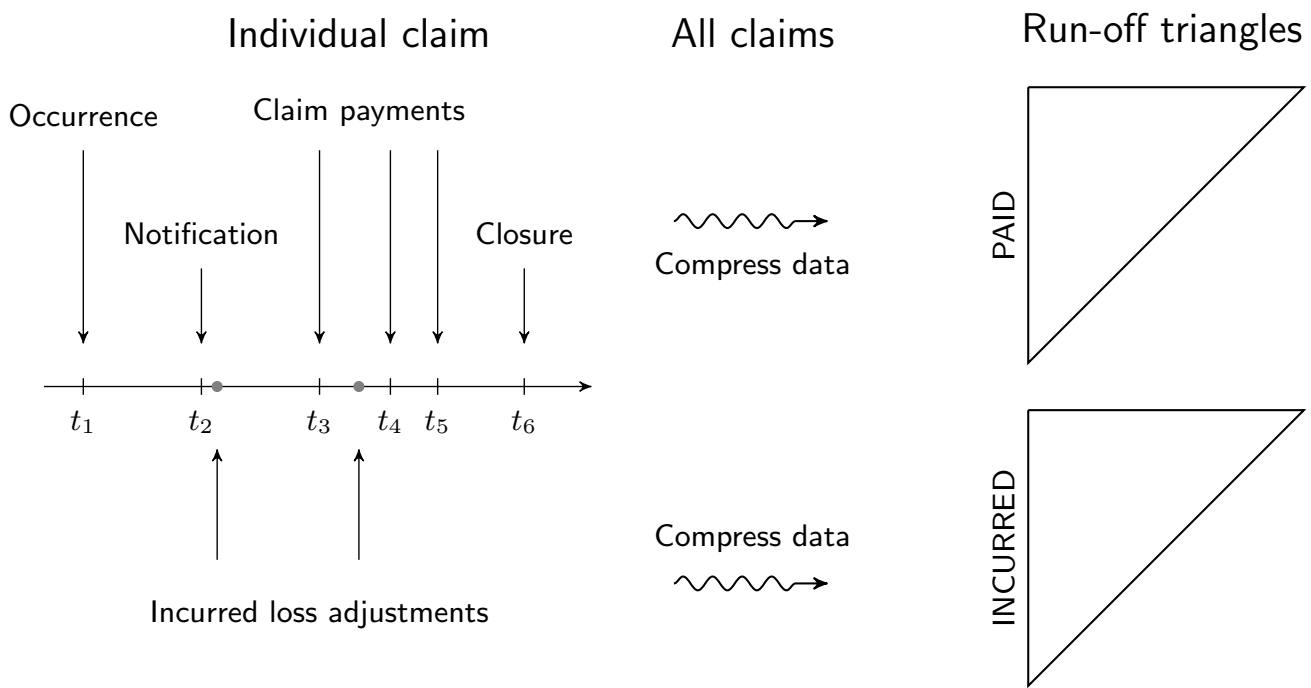

Figure 1: Time line representing the development of a non-life claim. The upper part illustrates claim payments over time, and the lower part shows incurred loss adjustments throughout the development of a claim. This information is then summarized in two run-off triangles: one for claim payments and one for incurred losses.

Reserving actuaries typically not only consider claim payments (the upper part in Figure 1) when evaluating reserves. They also analyze incurred losses (the lower part in Figure 1), especially in lines of business with large settlement delays and in reinsurance. Incurred losses are the sum of claim payments and case estimates. Such case estimates are set by an experienced case handler and express the expert's current estimate of the outstanding loss on an individual claim. Thus, case estimates might be adjusted or corrected throughout the development of a claim. Incurred losses therefore evolve, as Figure 1 illustrates. The incurred loss does not change when the case estimate is automatically adjusted by subtracting the paid amount (at the date of payment). Indeed, changes in claim payments and case estimates then annihilate and the incurred loss is unchanged. Incurred loss adjustments and claim payments should not necessarily occur at the same time. Typically, the initial case estimate (and thus: incurred loss) is determined by experts right after reporting of the claim. These features are illustrated in Figure 1.

In current actuarial practice both sources of information are summarized in separate triangles, see Figure 1 or Table 1 in Wüthrich and Merz (2010). The loss reserving actuary can either work with a single channel of information (e.g. only claim payments), or can use a paid-incurred method designed for triangles. Section 1 in Wüthrich and Merz (2010) is an excellent overview of the milestones in the literature covering such methods.

Recently, the necessity and appropriateness of the use of run-off triangles has been challenged. Building upon the fundamental work by Norberg (1993), Haastrup and Arjas (1996) and Norberg (1999), Antonio and Plat (2013) model the development of individual claims in continuous time. Drieskens et al. (2012), Rosenlund (2012) and Pigeon et al. (2013) work in discrete time and 
aggregate payments per time period (e.g. a development year), but keep the claim specific time line. Our paper extends the discrete time approach in Pigeon et al. (2013) and allows for consistent combination of two channels of information, namely claim payments and incurred losses. The relevance attached by practicing actuaries to incurred loss information motivates the importance of extending the tools for reserving with individual claims towards inclusion of incurred losses.

The paper is organized as follows. We introduce the statistical model in Section 2 and we present our main results in Section 3 . We devote Section 4 to the parameter estimation problem and we perform a case study in Section 5. Finally, we conclude in Section 6. Some technical developments are gathered in an Appendix, for the sake of completeness.

\section{Model specification and assumptions}

Our starting point is the availability of a data set with detailed information on the development of claim payments and incurred losses of individual claims. More specifically, the model uses the occurrence date, the reporting date, the date(s) and size(s) of claim payment(s) and incurred loss adjustment(s), and the closure date of the claim. We illustrate the available information in continuous time in the first three columns of Table 1. In column 1, ' $P$ ' refers to cumulative payments, and in column 2 ' $I$ ' is for incurred loss, as recorded on a MM/DD/YY basis.

\begin{tabular}{|c|c|c|c|c|}
\hline \multirow{2}{*}{\multicolumn{2}{|c|}{ Original information }} & \multirow[t]{2}{*}{ Date } & \multicolumn{2}{|c|}{ Our Notation } \\
\hline & & & Paid & Incurred \\
\hline \multicolumn{2}{|c|}{$\begin{array}{l}\text { Accident } \\
\text { Reporting }\end{array}$} & $\begin{array}{l}04 / 26 / 1997 \\
11 / 28 / 1997\end{array}$ & \multicolumn{2}{|c|}{$T=0$} \\
\hline \multicolumn{2}{|c|}{$\begin{array}{l}\text { Number of periods with event } \\
\text { after first one }\end{array}$} & & $\begin{array}{l}Q^{P}=1 \\
U^{P}=4\end{array}$ & $\begin{array}{l}Q^{I}=0 \\
U^{I}=4\end{array}$ \\
\hline$P=0$ & $I=4,402$ & $12 / 11 / 1997$ & & $I_{1}=4,402$ \\
\hline $\begin{array}{l}P=23 \\
P=83\end{array}$ & & $\begin{array}{l}09 / 30 / 1998 \\
11 / 12 / 1998 \\
\end{array}$ & $P_{1}=83$ & \\
\hline $\begin{array}{l}P=110 \\
P=134\end{array}$ & $I=6,217$ & $\begin{array}{l}01 / 07 / 1999 \\
10 / 16 / 1999 \\
\end{array}$ & $\begin{array}{c}N_{1}^{P}=1 \\
P_{2}=134\end{array}$ & $\begin{array}{c}N_{1}^{I}=2 \\
I_{2}=6,217\end{array}$ \\
\hline $\begin{array}{l}P=160 \\
P=550\end{array}$ & & $\begin{array}{l}01 / 21 / 2000 \\
11 / 08 / 2000\end{array}$ & $\begin{array}{c}N_{2}^{P}=1 \\
P_{3}=550\end{array}$ & \\
\hline $\begin{array}{l}P=578 \\
P=607\end{array}$ & $I=6,262$ & $\begin{array}{l}03 / 15 / 2001 \\
07 / 25 / 2001\end{array}$ & $\begin{array}{c}N_{3}^{P}=1 \\
P_{4}=607\end{array}$ & $\begin{array}{c}N_{2}^{I}=2 \\
I_{3}=6,262\end{array}$ \\
\hline $\begin{array}{l}P=1,867 \\
P=7,394\end{array}$ & $I=9,438$ & $\begin{array}{l}03 / 05 / 2002 \\
05 / 28 / 2002 \\
\end{array}$ & $\begin{array}{c}N_{4}^{P}=1 \\
P_{5}=7,394\end{array}$ & $\begin{array}{c}N_{3}^{I}=1 \\
I_{4}=9,438\end{array}$ \\
\hline$P=7,394$ & $I=7,394$ & $12 / 03 / 2003$ & & $\begin{array}{c}N_{4}^{I}=1 \\
I_{5}=7,394\end{array}$ \\
\hline & Closure & $12 / 03 / 2003$ & $N_{5}^{P}=1$ & $N_{5}^{I}=0$ \\
\hline
\end{tabular}

Table 1: Illustration of claim payments and incurred losses as recorded in continuous time for individual claims (first two columns). In columns 4 and 5 we demonstrate the discrete time notation used in this paper. The claim is a bodily injury claim from the data set analyzed in Section 5 . 


\section{$2.1 \quad$ Notation}

We leave the continuous time framework from Figure 1 and work in discrete time (e.g. with periods of one year). We denote the $k^{\text {th }}$ claim in a data base with $k$ and the number of claims from occurrence period $i$ by $K_{i}, i=1, \ldots, I$, where $I$ is the number of occurrence periods considered. Our discrete time framework has the same set up as in Pigeon et al. (2013), but is now extended to an additional source of information, namely the incurred losses. We specify the following:

Exposure. To model IBNR dynamics we should have a measure of exposure at our disposal, per period of occurrence, say $\theta_{i}$. We refer to Pigeon et al. (2013) for more details about exposure measures.

Reporting delay. $T_{k}$ is the reporting delay for claim $k$, i.e. the number of periods between the occurrence period of the claim and its notification to the insurance company. If the claim is reported during the period of occurrence, $T_{k}=0$.

Claim payments. A first source of information is claim payments (abbreviated with $P$ ).

P.1. $Q_{k}^{P}$ is the first payment delay; it is the number of periods between notification and the first payment $\neq 0$ for claim $k$;

P.2. $U_{k}^{P}$ is the number of period(s) with incremental claim payment $\neq 0$ after the first period with positive payment;

P.3. $N_{k j}^{P}$ is the delay between two periods with payment; it is the number of periods between payments $j$ and $j+1$. We use $N_{k, U_{k}^{P}+1}$ to denote the number of periods between the last payment and the settlement of the claim.

P.4. The first cumulative payment $(\neq 0)$ is denoted with $P_{k, 1}$. Consecutive cumulative payments are $P_{k, 2}, \ldots, P_{k, U_{k}^{P}+1}$. Since we focus on incremental claim payments $\neq 0$, $P_{k, j}$ differs from $P_{k, j-1}$.

Incurred losses. A second source of information is incurred losses (denoted with $I$ ), structured in a similar way as the claim payments information.

I.1. $Q_{k}^{I}$ is the initial case estimate delay; it is the number of periods between notification and the initialization of a case estimate for claim $k$. Usually, an initial case estimate is determined immediately after notification of the claim, as the first bullet in Figure 1 illustrates. However, depending on regulation, legislation and other external factors the initialization of the case estimate may require more time.

I.2. $U_{k}^{I}$ is the number of period(s) with adjustment of the incurred losses, after the period in which the initial case estimate is set.

I.3. $N_{k j}^{I}$ is the delay between two periods with adjustment of incurred losses. This is the number of periods between adjustments $j$ and $j+1$. We use $N_{k, U_{k}+1}$ to denote the number of periods between the last payment and the settlement of the claim.

I.4. The final incurred loss is denoted with $I_{k, U_{k}^{I}+1}$. Preceding incurred losses are $I_{k, 1}, \ldots, I_{k, U_{k}^{I}}$. Again, since we focus on adjustments to incurred losses $I_{k, j+1}$ differs from $I_{k, j}$. 
Table 1 illustrates this notation (in columns 4 and 5) for a particular bodily injury claim. Let $\tau$ denote the present moment, i.e. the moment of evaluation of reserves. We denote the collection of information on claim payments (over all claims), available at $\tau$, with $\mathcal{I}_{\tau}^{P}$. Similarly, $\mathcal{I}_{\tau}^{I}$ is the information on all incurred losses, as registered up to $\tau$. For the combined information we use $\mathcal{I}_{\tau}:=\mathcal{I}_{\tau}^{P} \cup \mathcal{I}_{\tau}^{I}$.

\subsection{Individual paid-incurred developments}

Given a claim $k$ and $U_{k}^{P}=u_{k}^{P}$, the cumulative payments $P_{k, j}$ are specified by the recursion

$$
P_{k, j}=P_{k, j-1} \cdot \lambda_{j-1}^{(k)} \quad j=2, \ldots, u_{k}^{P}+1,
$$

with initial value $P_{k, 1}$. As in Pigeon et al. (2013) the $\lambda_{j}^{(k)}$ 's are payment-to-payment link ratios. They are similar to chain-ladder development factors (see Mack (1993)), but $j$ now refers to a claim payment instead of development period. The claim payments information on claim $k$ is then summarized by the vector

$$
\left[\begin{array}{lllll}
P_{k, 1} & \lambda_{1}^{(k)} & \lambda_{2}^{(k)} & \ldots & \lambda_{u_{k}^{P}}^{(k)}
\end{array}\right]^{\prime}
$$

We combine the stream of information on payments with the information on incurred losses, as registered for claim $k$. Similar to the Paid-Incurred Chain ('PIC') approach (see Wüthrich and Merz (2010) ) for aggregate data, we impose the condition

$$
I_{k, u_{k}^{I}+1}=P_{k, u_{k}^{P}+1},
$$

given $U_{k}^{P}=u_{k}^{P}$ and $U_{k}^{I}=u_{k}^{I}$. This assumption guarantees that for each claim in the data set the ultimate loss coincides for claim payments and incurred losses, as it should be. The incurred losses then follow a backward recursion, namely

$$
I_{k, j-1}=I_{k, j} \cdot \gamma_{j-1}^{(k)} \quad j=2, \ldots, u_{k}^{I}+1,
$$

with initial value $I_{k, u_{k}^{I}+1}$. The $\gamma_{j}^{(k)}$ are link ratios which express the adjustments in the incurred losses for claim $k$. Again, we monitor these from adjustment-to-adjustment, instead of development-to-development (as the PIC does).

We illustrate the definition of payment-to-payment link ratios $\lambda^{(k)}$ and adjustment-to-adjustment factors $\gamma^{(k)}$ on the example developed in Table 1. For this particular example we obtain

$$
\begin{aligned}
& P_{k, 1}=83 \lambda_{1}^{(k)}=1.61 \quad \lambda_{2}^{(k)}=4.10 \quad \lambda_{3}^{(k)}=1.10 \quad \lambda_{4}^{(k)}=12.18 \\
& \gamma_{4}^{(k)}=1.28 \quad \gamma_{3}^{(k)}=0.66 \quad \gamma_{2}^{(k)}=0.99 \quad \gamma_{1}^{(k)}=0.71 .
\end{aligned}
$$

\subsection{Distributional assumptions}

We define $M=\max _{k}\left(u_{k}^{P}\right)+\max _{k}\left(u_{k}^{I}\right)+1$, a location vector $\boldsymbol{\mu}=\left[\begin{array}{ll}\mu_{1} & \ldots \mu_{M}\end{array}\right]^{\prime}$, a scale matrix

$$
\boldsymbol{\Sigma}=\left[\begin{array}{cccc}
\sigma_{11} & \sigma_{12} & \ldots & \sigma_{1 M} \\
\sigma_{21} & \sigma_{22} & \ldots & \sigma_{2 M} \\
\vdots & \vdots & \ddots & \vdots \\
\sigma_{M 1} & \sigma_{M 2} & \ldots & \sigma_{M M}
\end{array}\right]
$$


and a shape vector $\boldsymbol{\Delta}=\left[\begin{array}{lll}\Delta_{1} & \ldots & \Delta_{M}\end{array}\right]^{\prime}$. Let $\boldsymbol{\Omega}$ be a random vector combining both paid and incurred, on log scale

$$
\boldsymbol{\Omega}:=\left[\begin{array}{llllllll}
\log P_{1} & \log \lambda_{1} & \log \lambda_{2} & \ldots & \log \lambda_{\max _{k}\left(u_{k}^{P}\right)} & \log \gamma_{\max _{k}\left(u_{k}^{I}\right)} & \ldots & \log \gamma_{1}
\end{array}\right]^{\prime}
$$

For an ordered set $v=\left\{v_{1}, \ldots, v_{|v|}\right\},|v| \leqslant M$, we construct the random vector $\boldsymbol{\Omega}_{v}$ by selecting elements from $\boldsymbol{\Omega}$ corresponding to the subscripts collected in $v$, i.e. $\boldsymbol{\Omega}_{v}:=\left[\begin{array}{llll}\Omega_{v_{1}} & \Omega_{v_{2}} & \ldots & \Omega_{v_{|v|}}\end{array}\right]^{\prime}$. In a similar way, we construct the location vector $\boldsymbol{\mu}_{v}=\left[\begin{array}{lll}\mu_{v_{1}} & \ldots & \mu_{v_{\mid v}}\end{array}\right]^{\prime}$, the shape vector $\boldsymbol{\Delta}_{v}=\left[\begin{array}{llll}\Delta_{v_{1}} & \ldots & \Delta_{v_{|v|}}\end{array}\right]^{\prime}$ and the scale matrix

$$
\boldsymbol{\Sigma}_{v}=\left[\begin{array}{cccc}
\sigma_{v_{1} v_{1}} & \sigma_{v_{1} v_{2}} & \ldots & \sigma_{v_{1} v_{|v|}} \\
\vdots & \vdots & \ddots & \vdots \\
\sigma_{v_{|v|} v_{1}} & \sigma_{v_{|v|} v_{2}} & \ldots & \sigma_{v_{|v|} v_{|v|}}
\end{array}\right]
$$

We assume that the random vector $\boldsymbol{\Omega}_{v}$ follows a $|v|$-Multivariate Skew Normal $\left(\mathrm{MSN}_{|v|}\right)$ distribution with location vector $\boldsymbol{\mu}_{v}$, scale matrix $\boldsymbol{\Sigma}_{v}^{1 / 2}$ and shape vector $\boldsymbol{\Delta}_{v}$. Here, $\boldsymbol{\Sigma}_{v}^{1 / 2}$ is the square root of matrix $\left(\boldsymbol{\Sigma}_{v}^{1 / 2}\right)\left(\boldsymbol{\Sigma}_{v}^{1 / 2}\right)^{\prime}$ by Cholesky decomposition. The specification of the MSN distribution used in this paper is in Definition 11 (see Gupta and Chen (2004), Akdemir (2009), Akdemir and Gupta (2010) and Pigeon et al. (2013)). The corresponding moment generating function (mgf) is in Appendix A.

Definition 1 (MSN distribution). Let $\boldsymbol{\mu}=\left[\begin{array}{lll}\mu_{1} & \ldots & \mu_{l}\end{array}\right]^{\prime}$ be a vector of location parameters, $\boldsymbol{\Sigma} a$ $(l \times l)$ positive definite symmetric scale matrix and $\boldsymbol{\Delta}=\left[\Delta_{1} \ldots \Delta_{l}\right]^{\prime}$ a vector of shape parameters. The $(l \times 1)$ random vector $\mathbf{X}$ follows a $l$-Multivariate Skew Normal $\left(M S N_{l}\right)$ distribution if its density function is of the form

$$
\operatorname{MSN}_{l}\left(\mathbf{x} ; \boldsymbol{\mu}, \boldsymbol{\Sigma}^{1 / 2}, \boldsymbol{\Delta}\right)=\frac{2^{l}}{\operatorname{det}(\boldsymbol{\Sigma})^{1 / 2}} \cdot \phi_{l}\left(\boldsymbol{\Sigma}^{-1 / 2}(\mathbf{x}-\boldsymbol{\mu})\right) \cdot \prod_{j=1}^{l} \Phi\left(\Delta_{j} \mathbf{e}_{j}^{\prime} \boldsymbol{\Sigma}^{-1 / 2}(\mathbf{x}-\boldsymbol{\mu})\right),
$$

where $\mathbf{e}_{j}^{\prime}$ are the elementary vectors of the coordinate system $\mathbb{R}^{l}$ and

$$
\phi_{l}(\cdot)=\prod_{j=1}^{l} \phi(\cdot)
$$

where $\phi(\cdot)$ and $\Phi(\cdot)$ are the pdf and cdf of the standard Normal distribution, respectively.

The scale parameter $\boldsymbol{\Sigma}$ is not the usual variance-covariance matrix as in the Multivariate Normal distribution. Formula (6) is generally valid whatever the square root considered. In the remainder of the paper, we specify the matrix $\boldsymbol{\Sigma}^{1 / 2}$ instead of $\boldsymbol{\Sigma}$ because of the plurality of the square roots of $\boldsymbol{\Sigma}$.

For the time components (P.1-P.3) and (I.1-I.3) in Section 2.1 we use the same distributional assumptions as in Pigeon et al. (2013). We do not repeat the assumptions here, but explain them in the case study covered in Section 5. 


\section{Ultimate loss prediction}

As mentioned in Section 1, insurance companies distinguish RBNS claims and IBNR claims. In this paper, we divide RBNS claims into two subclasses: pure RBNS (or just RBNS in the following) and Reported But Not Paid ('RBNP'). The latter expression describes a RBNS claim for which no payments have been made yet and no case estimate has been determined yet. The total reserve amount comes from the prediction of the remaining run-off of RBNS claims, and the predictions for losses related to RBNP and IBNR claims.

For a claim $k$, we denote its development

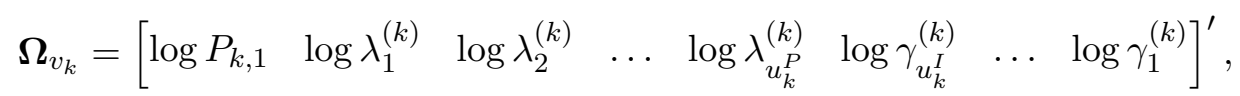

where the ordered set $v_{k}$ takes values $\left\{1, \ldots, u_{k}^{P}+1, \max _{k}\left(u_{k}^{P}\right)+1+\left(\max _{k}\left(u_{k}^{I}\right)-u_{k}^{I}\right), \ldots, M\right\}$. To relax notation we suppress the claim index in the propositions below.

Proposition 1. For a claim with development structure as in Section 2 and for given values $U^{P}=u^{P}$ and $U^{I}=u^{I}$, we denote $u^{P+I}:=u^{P}+u^{I}+1$, i.e. the length of the vector with combined $P$ and $I$ information, and have the following results.

(i) The vector

$$
\begin{aligned}
& \mathbf{D}=\left[\begin{array}{lllllll}
\ln P_{1} & \ln P_{2} & \ldots & \ln P_{u^{P}+1} & \ln I_{u^{I}} & \ldots & \ln I_{1}
\end{array}\right]^{\prime} \\
& =\mathbf{R} \boldsymbol{\Omega}_{v} \\
& =\left[\begin{array}{llll}
D_{1} & D_{2} & \ldots & D_{u^{P+I}}
\end{array}\right]^{\prime}
\end{aligned}
$$

with $\mathbf{R}$ the $u^{P+I} \times u^{P+I}$ matrix

$$
\mathbf{R}=\left[\begin{array}{ccccc}
1 & 0 & 0 & \ldots & 0 \\
1 & 1 & 0 & \ldots & 0 \\
1 & 1 & 1 & \ldots & 0 \\
\vdots & \vdots & \vdots & \ddots & \vdots \\
1 & 1 & 1 & \ldots & 1
\end{array}\right]
$$

follows a $M S N_{u^{P+I}}$ distribution with parameters $\mathbf{R} \boldsymbol{\mu}_{v}, \mathbf{R} \boldsymbol{\Sigma}_{v}^{1 / 2}$ and $\boldsymbol{\Delta}_{v}$.

(ii) The expected value of $P_{t}$ (with $\left.t=1, \ldots, u^{P}+1\right)$ is

$$
\begin{aligned}
& E\left[P_{t} \mid U^{P}=u^{P}, U^{I}=u^{I}\right] \\
= & 2^{u^{P+I}} \cdot \exp \left(\mathbf{R}_{[t,]} \boldsymbol{\mu}_{v}+0.5 \mathbf{R}_{[t,]}\left(\boldsymbol{\Sigma}_{v}^{1 / 2}\right)\left(\boldsymbol{\Sigma}_{v}^{1 / 2}\right)^{\prime} \mathbf{R}_{[t,]}^{\prime}\right) \cdot \prod_{j=1}^{u^{P+I}} \Phi\left(\frac{\Delta_{v, j}\left(\left(\boldsymbol{\Sigma}_{v}^{1 / 2}\right)^{\prime} \mathbf{R}_{[t,]}^{\prime}\right)_{j}}{\sqrt{1+\Delta_{v, j}^{2}}}\right),
\end{aligned}
$$

with $\mathbf{R}_{[t,]}$ the $t^{\text {th }}$ row of $\mathbf{R}$. The expected value of $I_{t}$ (with $t=1, \ldots, u^{I}$, and $t^{\star}=u^{P+I}-$ 


$$
\begin{aligned}
t+1) & i s \\
& E\left[I_{t} \mid U^{P}=u^{P}, U^{I}=u^{I}\right] \\
= & 2^{u^{P+I}} \cdot \exp \left(\mathbf{R}_{\left[t^{\star},\right]} \boldsymbol{\mu}_{v}+0.5 \mathbf{R}_{\left[t^{\star},\right]}\left(\boldsymbol{\Sigma}_{v}^{1 / 2}\right)\left(\boldsymbol{\Sigma}_{v}^{1 / 2}\right)^{\prime} \mathbf{R}_{\left[t^{\star},\right]}^{\prime}\right) \cdot \prod_{j=1}^{u^{P+I}} \Phi\left(\frac{\Delta_{v, j}\left(\left(\boldsymbol{\Sigma}_{v}^{1 / 2}\right)^{\prime} \mathbf{R}_{\left[t^{\star},\right]}^{\prime}\right)_{j}}{\sqrt{1+\Delta_{v, j}^{2}}}\right) .
\end{aligned}
$$

(iii) The covariance between $e^{D_{i}}$ and $e^{D_{j}}$ (with $i, j=1, \ldots, u^{P+I}$ ) is

$$
\begin{aligned}
& \operatorname{Cov}\left[e^{D_{i}}, e^{D_{j}} \mid U^{P}=u^{P}, U^{I}=u^{I}\right] \\
= & 2^{u^{P+I}} \cdot \exp \left(\mathbf{t}_{i+j}^{\prime} \mathbf{R} \boldsymbol{\mu}_{v}+0.5 \mathbf{t}_{i+j}^{\prime} \mathbf{R}\left(\boldsymbol{\Sigma}_{v}^{1 / 2}\right)\left(\boldsymbol{\Sigma}_{v}^{1 / 2}\right)^{\prime} \mathbf{R}^{\prime} \mathbf{t}_{i+j}\right) \cdot \prod_{n=1}^{u^{P+I}} \Phi\left(\frac{\Delta_{v, n}\left(\left(\boldsymbol{\Sigma}_{v}^{1 / 2}\right)^{\prime} \mathbf{R}^{\prime} \mathbf{t}_{i+j}\right)_{n}}{\sqrt{1+\Delta_{v, n}^{2}}}\right) \\
& -2^{2\left(u^{P+I}\right)} \cdot \exp \left(\mathbf{R}_{[i,]} \boldsymbol{\mu}_{v}+\mathbf{R}_{[j,]} \boldsymbol{\mu}_{v}+0.5\left(\mathbf{R}_{[i,]}\left(\boldsymbol{\Sigma}_{v}^{1 / 2}\right)\left(\boldsymbol{\Sigma}_{v}^{1 / 2}\right)^{\prime} \mathbf{R}_{[i,]}^{\prime}+\mathbf{R}_{[j,]}\left(\boldsymbol{\Sigma}_{v}^{1 / 2}\right)\left(\boldsymbol{\Sigma}_{v}^{1 / 2}\right)^{\prime} \mathbf{R}_{[j,]}^{\prime}\right)\right. \\
& \times \prod_{n=1}^{u^{P+I}} \Phi\left(\frac{\Delta_{v, n}\left(\left(\boldsymbol{\Sigma}_{v}^{1 / 2}\right)^{\prime} \mathbf{R}_{[i,]}^{\prime}\right)_{n}}{\sqrt{1+\Delta_{v, n}^{2}}}\right) \cdot \prod_{n=1}^{u^{P+I}} \Phi\left(\frac{\Delta_{v, n}\left(\left(\boldsymbol{\Sigma}_{v}^{1 / 2}\right)^{\prime} \mathbf{R}_{[j,]}^{\prime}\right)_{n}}{\sqrt{1+\Delta_{v, n}^{2}}}\right),
\end{aligned}
$$

with $\mathbf{t}_{i+j}$ is the sum of two $u^{P+I}$ column vectors with zero elements except for ' 1 ' at its $i^{\text {th }}$, respectively $j^{\text {th }}$, row.

The proof of Proposition 1 follows in Appendix B. From this Proposition, we obtain expected values and covariances for cumulative paid and incurred amounts.

Proposition 2. For a claim with development structure as in Section 2 and for given values $U^{P}=u^{P}$ and $U^{I}=u^{I}$, we denote $u^{P+I}:=u^{P}+u^{I}+1$. Let 'vo' be the set of subscripts corresponding to the observed part of the random vector $\boldsymbol{\Omega}_{v}$, ' $v f$ ' the complementary set and $v^{\prime}:=\{v o, v f\}$. In both vectors, subscripts are in increasing order, e.g. vo $o_{1}<v o_{2}<\ldots$ Let $t^{P}$ be the number of periods with observed partial payments and $t^{I}$ the number of periods with observed adjustments in incurred losses.

The random vector $\boldsymbol{\Omega}_{v^{\prime}}$ follows a MSN u $^{P+I}$ distribution with parameters $\boldsymbol{\mu}_{v^{\prime}}, \boldsymbol{\Sigma}_{v^{\prime}}^{1 / 2}$ and $\boldsymbol{\Delta}_{v^{\prime}}$. We define

$$
\boldsymbol{\mu}_{v^{\prime}}:=\left[\begin{array}{c}
\tilde{\boldsymbol{\mu}}_{o} \\
\tilde{\boldsymbol{\mu}}_{f}
\end{array}\right], \quad \boldsymbol{\Sigma}_{v^{\prime}}^{1 / 2}:=\left[\begin{array}{cc}
\tilde{\boldsymbol{\Sigma}}_{o o} & \mathbf{0} \\
\tilde{\boldsymbol{\Sigma}}_{f o} & \tilde{\boldsymbol{\Sigma}}_{f f}
\end{array}\right], \quad \boldsymbol{\Delta}_{v^{\prime}}:=\left[\begin{array}{c}
\tilde{\boldsymbol{\Delta}}_{o} \\
\tilde{\boldsymbol{\Delta}}_{f}
\end{array}\right]
$$

$\tilde{\boldsymbol{\mu}}_{o}$ and $\tilde{\boldsymbol{\Delta}}_{o}$ are $\mid$ vo $\mid$-column vectors, and $\tilde{\boldsymbol{\mu}}_{f}$ and $\tilde{\boldsymbol{\Delta}}_{f}$ are $\mid$ vf $\mid$-column vectors. $\tilde{\boldsymbol{\Sigma}}_{o o}$ is a $\mid$ vo $|\times|$ vo $\mid$ lower triangular matrix with positive diagonal elements and $\tilde{\boldsymbol{\Sigma}}_{f f}$ is a $|v f| \times|v f|$ lower triangular matrix with positive diagonal elements. Denote $\boldsymbol{\mu}^{\star}:=\tilde{\boldsymbol{\mu}}_{f}+\tilde{\boldsymbol{\Sigma}}_{f o} \tilde{\boldsymbol{\Sigma}}_{o o}^{-1}\left(\boldsymbol{\omega}_{v o}-\tilde{\boldsymbol{\mu}}_{o}\right), \boldsymbol{\Sigma}^{\star}:=\tilde{\boldsymbol{\Sigma}}_{f f}$ and $\boldsymbol{\Delta}^{\star}:=\tilde{\boldsymbol{\Delta}}_{f}$. The expected value of $P_{t^{P}+s}$ with $s=1, \ldots, u^{P}+1-t^{P}$, conditional on the observed stream of combined $(P, I)$ information in $\boldsymbol{\Omega}_{v o}$, is

$$
\begin{aligned}
& E\left[P_{t^{P}+s} \mid \boldsymbol{\Omega}_{v o}=\boldsymbol{\omega}_{v o}, U^{P}=u^{P}, U^{I}=u^{I}\right] \\
= & \exp \left(\sum_{j=1}^{t^{P}} \omega_{j}\right) \cdot 2^{|v f|} \exp \left(\mathbf{t}_{s}^{\star} \boldsymbol{\mu}^{\star}+0.5 \mathbf{t}_{s}^{\star}\left(\left(\boldsymbol{\Sigma}^{\star}\right)^{1 / 2}\right)\left(\left(\boldsymbol{\Sigma}^{\star}\right)^{1 / 2}\right)^{\prime}\left(\mathbf{t}_{s}^{\star}\right)^{\prime}\right) \cdot \prod_{j=1}^{|v f|} \Phi\left(\frac{\Delta_{j}^{\star}\left(\left(\left(\boldsymbol{\Sigma}^{\star}\right)^{1 / 2}\right)^{\prime}\left(\mathbf{t}_{s}^{\star}\right)^{\prime}\right)_{j}}{\sqrt{1+\left(\Delta_{j}^{\star}\right)^{2}}}\right) .
\end{aligned}
$$


Hereby $\mathbf{t}_{s}^{\star}:=\left[\begin{array}{lllllll}1 & \ldots & 1 & 1 & 0 & \ldots & 0\end{array}\right]$ is a $|v f|$-row vector with 1 at position $1, \ldots, s$ and 0 elsewhere. Similar expressions for $E\left[I_{t^{I}+s} \mid \boldsymbol{\Omega}_{v o}=\boldsymbol{\omega}_{v o}, U^{P}=u^{P}, U^{I}=u^{I}\right]$ are directly available.

The proof of Proposition 2 follows in Appendix C. The conditional expected value including past information on claim payments or incurred losses only follows from Proposition 2 by choosing an appropriate subset of $v$. Using the results from Propositions 1 and 2, an expression for the total IBNR, RBNP and RBNS reserve easily follows.

Proposition 3 (Best estimates for ultimate RBNS, RBNP and IBNR losses). Let $\mathcal{I}_{\tau}$ denote the combined information available for all claims in the data set at evaluation moment $\tau$, and $\mathcal{I}_{k, \tau}$ the specific information available for claim $k$. The latter is the observed part of the development of claim k, i.e. $\boldsymbol{\Omega}_{v_{k}}$ (see Proposition 2). Using the development structure from Section 2, we obtain the following expressions for the ultimate IBNR, RBNP and RBNS losses.

(i) The expected value of the total outstanding IBNR and RBNP reserves, respectively, is

$$
E\left[I B N R \mid \mathcal{I}_{\tau}\right] \text { versus } E\left[R B N P \mid \mathcal{I}_{\tau}\right]=(x) \cdot E_{U^{P}, U^{I}}\left[E\left[P_{U^{P}+1} \mid U^{P}, U^{I}\right]\right],
$$

where $(x)$ should be replaced with $E\left[K_{I B N R}\right]$, the expected number of IBNR claims for the given data set, in case of IBNR reserves, and with $k_{R B N P}$, the observed number of open claims without payment and without initial case estimate, in case of RBNP reserves. As in Pigeon et al. (2013) the occurrence of IBNR claims is driven by a thinned Poisson process, where thinning is based on the delay in reporting the claim to the insurance company. $P_{U^{P}+1}$ is the random variable representing the ultimate loss of a claim, and $E\left[P_{U^{P}+1} \mid U^{P}, U^{I}\right]$ follows from Proposition 1 (ii).

(ii) The expected value of the ultimate RBNS loss is

$$
E\left[R B N S \mid \mathcal{I}_{\tau}\right]=\sum_{k=1}^{K_{R B N S}} E_{U^{P}, U^{I}}\left[E\left[P_{k, U_{k}^{P}+1} \mid \mathcal{I}_{k, \tau}=\boldsymbol{\Omega}_{v o_{k}}, U_{k}^{P}, U_{k}^{I}\right]\right]
$$

where the sum runs over all RBNS claims in the data set. $K_{R B N S}$ is the observed number of $R B N S$ claims for the data at hand. For each RBNS claim $E\left[P_{k, U_{k}^{P}+1} \mid \mathcal{I}_{k, \tau}=\boldsymbol{\Omega}_{v_{k}}, U_{k}^{P}, U_{k}^{I}\right]$ follows from Proposition 2 . The corresponding best estimate of the RBNS reserve then follows by subtracting the most recent observed cumulative claim amount.

\section{Parameter estimation}

Parameter estimation is performed as in Pigeon et al. (2013). We use maximum likelihood for estimating parameters of the distributions describing time dynamics (i.e. the random variables $U, Q$ and $N$ in Section 2.1). To estimate shape parameters, we follow an iterative procedure inspired by Akdemir (2009). We take the following steps: $(i)$ start with initial values for shape parameters; (ii) obtain estimates for location and scale parameters by the method of moments; (iii) obtain updated estimates for shape parameters by the maximum product of spacings method (see Cheng and Amin (1983) and Ranneby (1984)); and (iv) repeat steps (ii) and (iii) until convergence. With the resulting shape parameters held fixed, we obtain location and scale parameters by applying maximum likelihood. In the likelihood optimization, numerical approximation of the Hessian matrix is used to estimate standard errors for location and scale parameters. We perform data manipulations and likelihood optimization with R. A sample $R$ program illustrating the estimation procedure is available from the authors. 


\section{Case study}

\subsection{The data}

Background. We use data from a portfolio of general liability insurance policies for private individuals. The claim payments from this data source have been analyzed in Antonio and Plat (2013) and Pigeon et al. (2013). However, the information on incurred losses has not been considered before. Our analysis uses claim payments and incurred losses registered between January 1, 1997 and December 31, 2005. For these claims, we have access to paid and incurred losses registered in years 2006, 2007 and 2008. We will use this information to evaluate our results. Two types of payments are in the data set: Bodily Injury (BI) and Material Damage (MD). We demonstrate the paid-incurred individual reserving method using BI claims. Corresponding run-off triangles are in Figures 2 (claim payments) and 3 (incurred losses).

$\begin{array}{rrrrrrrrrr} & 1 & 2 & 3 & 4 & 5 & 6 & 7 & 8 & 9 \\ 1997 & 271148 & 905934 & 1284478 & 1861430 & 2473545 & 2631995 & 2786279 & 3196535 & 3210059 \\ 1998 & 213628 & 712468 & 1048113 & 1424123 & 1735178 & 1802001 & 2018831 & 2114466 & \text { NA } \\ 1999 & 255561 & 870035 & 1325213 & 1640168 & 1943254 & 2290108 & 2452256 & \text { NA } & \text { NA } \\ 2000 & 260538 & 905787 & 1421300 & 2016922 & 2508790 & 2966609 & \text { NA } & \text { NA } & \text { NA } \\ 2001 & 458840 & 1418720 & 2077303 & 2754281 & 3302786 & \text { NA } & \text { NA } & \text { NA } & \text { NA } \\ 2002 & 318890 & 1015278 & 1663586 & 2212191 & \text { NA } & \text { NA } & \text { NA } & \text { NA } & \text { NA } \\ 2003 & 298100 & 1216496 & 1964751 & \text { NA } & \text { NA } & \text { NA } & \text { NA } & \text { NA } & \text { NA } \\ 2004 & 328135 & 1371792 & \text { NA } & \text { NA } & \text { NA } & \text { NA } & \text { NA } & \text { NA } & \text { NA } \\ 2005 & 554960 & \text { NA } & \text { NA } & \text { NA } & \text { NA } & \text { NA } & \text { NA } & \text { NA } & \text { NA }\end{array}$

Figure 2: Bodily Injury: cumulative triangle with claim payments, aggregated by occurrence and development year.

$\begin{array}{rrrrrrrrrr} & 1 & 2 & 3 & 4 & 5 & 6 & 7 & 8 & 9 \\ 1997 & 3031512 & 3841628 & 3941695 & 3943014 & 3951643 & 4236078 & 4108628 & 3988837 & 3824533 \\ 1998 & 1677016 & 1916667 & 2031341 & 2310552 & 2829381 & 2715373 & 2507187 & 2599724 & \text { NA } \\ 1999 & 2119737 & 2484931 & 2725846 & 3329335 & 3331172 & 3057421 & 3096374 & \text { NA } & \text { NA } \\ 2000 & 1946812 & 3151446 & 3929432 & 3966003 & 4156290 & 4595953 & \text { NA } & \text { NA } & \text { NA } \\ 2001 & 3059369 & 4221567 & 4745409 & 5100208 & 5584066 & \text { NA } & \text { NA } & \text { NA } & \text { NA } \\ 2002 & 4320019 & 4215660 & 5425686 & 5930939 & \text { NA } & \text { NA } & \text { NA } & \text { NA } & \text { NA } \\ 2003 & 2713203 & 3796245 & 3904498 & \text { NA } & \text { NA } & \text { NA } & \text { NA } & \text { NA } & \text { NA } \\ 2004 & 3461298 & 4353255 & \text { NA } & \text { NA } & \text { NA } & \text { NA } & \text { NA } & \text { NA } & \text { NA } \\ 2005 & 3935140 & \text { NA } & \text { NA } & \text { NA } & \text { NA } & \text { NA } & \text { NA } & \text { NA } & \text { NA }\end{array}$

Figure 3: Bodily Injury: cumulative triangle with incurred losses, aggregated by occurrence and development year.

Incurred losses and claim payments coincide at settlement of the claim, as required by condition (3). The latest observed cumulative paid amount is $20,149,870$ euro (in total), while the most recent total incurred loss is $37,824,482$ euro. This is the sum of the numbers on the diagonal in Figures 2 and 3, respectively. Thus, the claim experts' estimate of the total outstanding loss is $37,824,482-20,149,870=17,674,612$ euro, as evaluated at the end of 2005 .

Descriptive statistics. The data set consists of 7,367 reported BI claims of which 6,197 claims are closed at the time of evaluation. We present descriptive statistics for closed claims with positive payments in Table 2. We illustrate dependence between the components of development vector $\boldsymbol{\Omega}(\sqrt[5 p)]{)}$ in Figure 5 . 


\begin{tabular}{lllllll}
\hline Variables & Mean & Median & s.e. & Minimum & Maximum & $\begin{array}{l}\text { Number of } \\
\text { Observations }\end{array}$ \\
\hline$P_{1}$ & 1,190 & 397 & 3,272 & 6.58 & 162,300 & 3,700 \\
$\lambda_{1}$ & 10.78 & 3.30 & 32.99 & 1.00 & 667.70 & 1,245 \\
$\lambda_{2}$ & 4.81 & 1.99 & 10.97 & 1.00 & 131.80 & 343 \\
$\lambda_{3}$ & 2.67 & 1.74 & 2.22 & 0.51 & 12.57 & 116 \\
$\lambda_{4}$ & 2.28 & 1.65 & 1.89 & 0.96 & 12.19 & 51 \\
\hline$\gamma_{1}$ & 8.71 & 2.21 & 45.00 & 0.001 & 1,528 & 1,958 \\
$\gamma_{2}$ & 6.04 & 1.31 & 58.61 & 0.8 & 1,269 & 483 \\
$\gamma_{3}$ & 1.79 & 1.14 & 3.76 & 0.02 & 42.84 & 131 \\
$\gamma_{4}$ & 3.48 & 1.02 & 13.25 & 0.46 & 80.62 & 36 \\
\hline
\end{tabular}

Table 2: Descriptive statistics for closed claims: first payment and development factors $\lambda_{j}$ and $\gamma_{j}$ with $j \leqslant 4$ for BI claims.

\subsection{Estimation results}

Occurrence of claims. We first model reporting delays, in a similar way as Pigeon et al. (2013). We investigate the use of a discrete distribution combined with some degenerate components, specified as

$$
f_{1}(t ; \boldsymbol{\nu})=\sum_{s=0}^{p} \nu_{s} \cdot I_{s}(t)+\left(1-\sum_{s=0}^{p} \nu_{s}\right) f_{T \mid T>p}(t),
$$

where $I_{s}(t)=1$ for reporting in period $s$ after the period of occurrence and 0 otherwise. $f(t)$ is the probability mass function of a discrete distribution with parameter(s) $\nu_{p+1}, \ldots, \nu_{p+q}$. We consider the use of a Geometric, Binomial, Poisson and Negative Binomial distribution for $f(\cdot)$, combined with $p+1$ degenerate components $(p=-1,0,1,2,3)$. Our preferred specification (using BIC) is a Geometric distribution, with a degenerate component for reporting at ' 0 ' and ' 1 ' periods after occurrence. Corresponding parameter estimates are in Table 3.

The number of claims from occurrence period $i$, say $K_{i}$, follows a Poisson distribution with intensity $\theta \cdot w(i) . w(i)$ is the exposure registered for occurrence period $i$. However, since we only observe reported claims, the Poisson distribution should be thinned in the following way

$$
\theta \cdot w(i) \cdot F_{1}\left(r_{i}^{\star}-1 ; \boldsymbol{\nu}\right) .
$$

Evaluation then takes place $r_{i}^{\star}$ periods after occurrence period $i$, at the beginning of this period. $F_{1}(\cdot ; \boldsymbol{\nu})$ is the distribution function for reporting delay with unknown parameters $\boldsymbol{\nu}$ corresponding to the probability mass function (14). We calibrate this thinned Poisson process to the number of reported claims per occurrence period, and obtain $\hat{\theta}=0.8117$ (s.e. 0.0148).

Time dynamics. For the other discrete random variables describing the time dynamics of a claim (i.e. $U^{P}, U^{I}, Q^{P}, Q^{I}, N^{P}$ and $N^{I}$, see Section 2.1), we also consider mixtures of a discrete distribution with degenerate components. Again, our preferred specification is a Geometric distribution with degenerate components. Corresponding results for $U^{P}$ and $U^{I}$, i.e. the number of periods with payment $\neq 0$ (after the first one) and the number of periods with adjustment of incurred loss (after the case estimate initialization), are in Table 3. Since this case study reports results for ultimate losses, we only show estimation results for the distribution of $T, U^{P}$ and $U^{I}$. 


\begin{tabular}{ccc}
\hline $\begin{array}{c}\text { Reporting delay } \\
(T ; \boldsymbol{\nu})\end{array}$ & $\begin{array}{c}\text { \# Claim payments } \\
\left(U^{P} ; \boldsymbol{\beta}\right)\end{array}$ & $\begin{array}{c}\text { \# Incurred loss } \\
\text { adjustments } \\
\left(U^{I} ; \boldsymbol{\gamma}\right) \\
(\text { s.e. })\end{array}$ \\
\hline 0.8997$)$ & 0.5259 & 0.3586 \\
$(0.005)$ & $(0.010)$ & $(0.008)$ \\
0.0790 & 0.2325 & 0.3771 \\
$(0.004)$ & $(0.007)$ & $(0.008)$ \\
0.5271 & 0.0761 & 0.1178 \\
$(0.062)$ & $(0.005)$ & $(0.006)$ \\
& 0.2053 & 0.2606 \\
& $(0.018)$ & $(0.028)$ \\
\hline
\end{tabular}

Table 3: Parameter estimates and standard errors for a Geometric distribution with degenerate components, as used for $\left\{T_{k}\right\},\left\{U_{k}^{P}\right\}$ and $\left\{U_{k}^{I}\right\}$ random variables in Section 2.1 .

MSN distribution for paid and incurred development vector. We consider two distributional specifications for the development vector $\boldsymbol{\Omega}$ (see (5)): the MSN distribution on the one hand and the Multivariate Normal (MN) (or, MSN with $\boldsymbol{\Delta}=\mathbf{0}$ ) distribution on the other hand. In the estimation process we calibrate the development vector corresponding to claim payments (i.e. $\left.\log P_{1}, \log \lambda_{1}, \log \lambda_{2}, \ldots, \log \lambda_{\max _{k}\left(u_{k}^{P}\right)}\right)$ with a maximum dimension of 5 . We estimate the development vector corresponding to incurred losses (i.e. $\log \gamma_{\max _{k}\left(u_{k}^{I}\right)}, \ldots, \log \gamma_{1}$ ) with a maximum dimension of 4 . This is motivated by the fact that very few claims have more than 5 periods with incremental payment or more than 4 periods with adjustment in incurred loss (see the descriptive statistics in Table 2). Thus, for the given data, the vector $\boldsymbol{\mu}$ has dimension $(9 \times 1)$, the matrix $\boldsymbol{\Sigma}$ has dimension $(9 \times 9)$ and the vector $\boldsymbol{\Delta}$ has dimension $(9 \times 1)$. When the development process of a claim uses less development factors, appropriate sub-vectors and sub-matrices are used in the likelihood, as discussed in Section 2.3 .

We visualize the empirical correlation matrix corresponding to $\Omega$ with a heat map in Figure 4 .

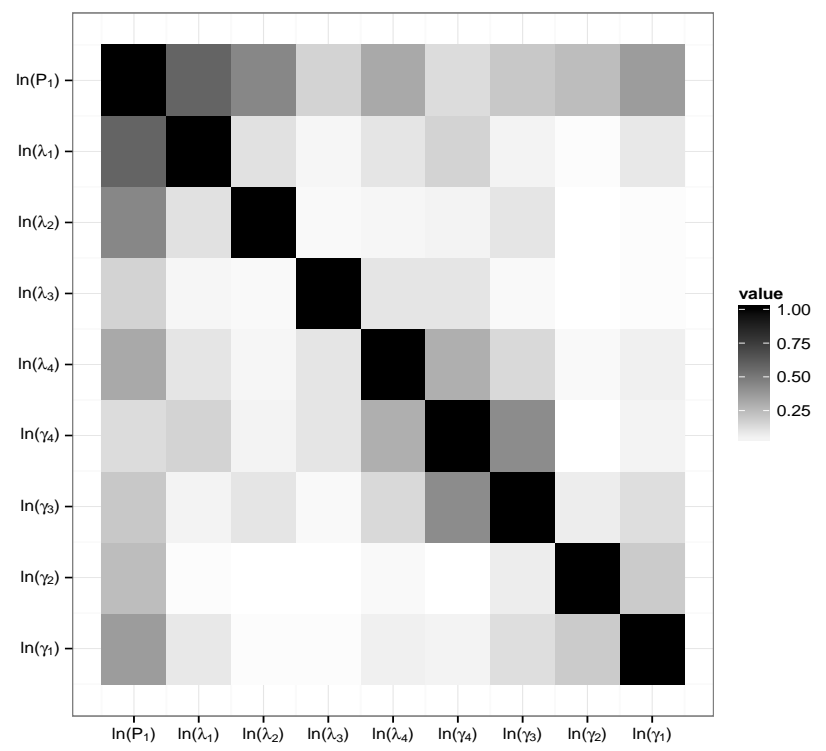

Figure 4: Empirical correlation matrix of $\boldsymbol{\Omega}$, visualized as heat map (in absolute value). 
Figure 4 motivates the following structures for the matrix $\boldsymbol{\Sigma}$ : $(i)$ a diagonal structure $\left(\boldsymbol{\Sigma}_{A}\right),(i i)$ dependence between the first payment and all development factors $\left(\boldsymbol{\Sigma}_{B}\right)$ and (iii) dependence between the first payment and all development factors and between two successive elements of the vector $\boldsymbol{\Omega}\left(\boldsymbol{\Sigma}_{C}\right)$ :

$\boldsymbol{\Sigma}_{A}=\left[\begin{array}{ccccc}\sigma_{11} & 0 & 0 & \ldots & 0 \\ 0 & \sigma_{22} & 0 & \ldots & 0 \\ 0 & 0 & \sigma_{33} & \ldots & 0 \\ \vdots & \vdots & \ddots & \vdots & \\ 0 & 0 & 0 & \ldots & \sigma_{99}\end{array}\right], \quad \boldsymbol{\Sigma}_{B}=\left[\begin{array}{ccccc}\sigma_{11} & \sigma_{12} & \sigma_{13} & \ldots & \sigma_{19} \\ \sigma_{12} & \sigma_{22} & 0 & \ldots & 0 \\ \sigma_{13} & 0 & \sigma_{33} & \ldots & 0 \\ \vdots & \vdots & \ddots & \vdots & \\ \sigma_{19} & 0 & 0 & \ldots & \sigma_{99}\end{array}\right], \quad \boldsymbol{\Sigma}_{C}=\left[\begin{array}{cccccc}\sigma_{11} & \sigma_{12} & \sigma_{13} & \ldots & 0 & \sigma_{19} \\ \sigma_{12} & \sigma_{22} & \sigma_{23} & \ldots & 0 & 0 \\ \sigma_{13} & \sigma_{23} & \sigma_{33} & \ldots & 0 & 0 \\ \vdots & \vdots & \ddots & \vdots & \vdots & \\ \sigma_{18} & 0 & 0 & \ldots & \sigma_{88} & \sigma_{89} \\ \sigma_{19} & 0 & 0 & \ldots & \sigma_{89} & \sigma_{99}\end{array}\right]$.

Table 4 reports the Akaike and Bayesian Information Criterion (AIC, BIC), the number of parameters used and the realized log-likelihood for the MSN and MN distributions with scale matrix specified as $\boldsymbol{\Sigma}_{A}, \boldsymbol{\Sigma}_{B}$ or $\boldsymbol{\Sigma}_{C}$. Based on these statistics, we prefer the MSN to the MN assumption, and the preferred structure for the scale matrix is $\boldsymbol{\Sigma}_{B}$. Table 5 shows corresponding

\begin{tabular}{llllll}
\hline & \# parameters & $\begin{array}{l}\text { Scale } \\
\text { matrix }\end{array}$ & -ll & AIC & BIC \\
\hline \multirow{3}{*}{ MSN } & $18+9=27$ & $\boldsymbol{\Sigma}_{A}$ & 13,387 & 26,828 & 26,996 \\
& $26+9=35$ & $\boldsymbol{\Sigma}_{B}$ & 12,612 & 25,294 & $\mathbf{2 5 , 5 1 2}$ \\
& $33+9=42$ & $\boldsymbol{\Sigma}_{C}$ & 12,599 & 25,282 & 25,543 \\
\hline \multirow{4}{*}{ MN } & 18 & $\boldsymbol{\Sigma}_{A}$ & 13,421 & 26,878 & 26,989 \\
& 26 & $\boldsymbol{\Sigma}_{B}$ & 12,891 & 25,834 & 25,996 \\
& 33 & $\boldsymbol{\Sigma}_{C}$ & 12,868 & 25,802 & 26,007 \\
\hline
\end{tabular}

Table 4: A comparison of MSN versus MN specification for the logarithm of the development vector $\boldsymbol{\Omega}$ (5) with different structures for $\boldsymbol{\Sigma}$.

parameter estimates. A selection of examples of observed data and fitted density contours are in Figure 5 .

\subsection{Prediction results}

\subsubsection{Analtyical best estimates}

We obtain analytical results for the expected value of total RBNS, RBNP and IBNR reserves using the result from Proposition 3, where unknown parameters are replaced with estimates. These expressions evaluate claims until settlement, even if this takes place beyond the boundary of the triangle. Table 6 displays these analytical results for the Bodily Injury case study, using the MSN and MN distribution for the development vector.

\subsubsection{Simulation results}

IBNR and RBNP reserves. For each occurrence period, we simulate the number of IBNR claims from a thinned Poisson process (see (15)), where thinning is based on reporting delay (see (14)). For each IBNR claim $k$, we simulate the number of period(s) with claim payments $U_{k}^{P}+1$, as well as the number of periods with incurred loss adjustment $U_{k}^{I}+1$. Consequently, we simulate the development vector $\Omega_{k}$ (see (5)). As such, we develop a claim until settlement 


\begin{tabular}{llll}
\hline $\begin{array}{l}\text { Location } \\
\boldsymbol{\mu} \text { (s.e.) }\end{array}$ & \multicolumn{2}{c}{ Scale } & Shape \\
$\boldsymbol{\Sigma}^{1 / 2}$ & $\boldsymbol{\Delta}$ \\
\hline$\mu_{1}=4.81$ & $\sigma_{11}=3.78$ & $\sigma_{12}=-1.36$ & $\Delta_{1}=1.37$ \\
$(0.03)$ & $\sigma_{22}=1.82$ & $\sigma_{13}=-0.70$ & $\Delta_{2}=1.56$ \\
$\mu_{2}=1.03$ & $\sigma_{33}=0.93$ & $\sigma_{14}=-0.26$ & $\Delta_{3}=1.07$ \\
$(0.03)$ & $\sigma_{44}=0.97$ & $\sigma_{15}=-0.32$ & $\Delta_{4}=4.43$ \\
$\mu_{3}=0.79$ & $\sigma_{55}=0.44$ & $\sigma_{16}=-0.18$ & $\Delta_{5}=1.09$ \\
$(0.05)$ & $\sigma_{66}=1.00$ & $\sigma_{17}=-0.20$ & $\Delta_{6}=2.66$ \\
$\mu_{4}=0.15$ & $\sigma_{77}=0.73$ & $\sigma_{18}=-0.58$ & $\Delta_{7}=0.49$ \\
$(0.06)$ & $\sigma_{88}=1.35$ & $\sigma_{19}=-1.77$ & $\Delta_{8}=-0.39$ \\
$\mu_{5}=0.51$ & $\sigma_{99}=4.13$ & & $\Delta_{9}=-1.76$ \\
$(0.09)$ & & & \\
$\mu_{6}=-0.32$ & & & \\
$(0.11)$ & & & \\
$\mu_{7}=-0.05$ & & & \\
$(0.07)$ & & & \\
$\mu_{8}=0.89$ & & & \\
$(0.05)$ & & & \\
$\mu_{9}=2.96$ & & & \\
$(0.04)$ & & & \\
\hline
\end{tabular}

Table 5: Parameter estimates for the MSN distribution used in the case study.
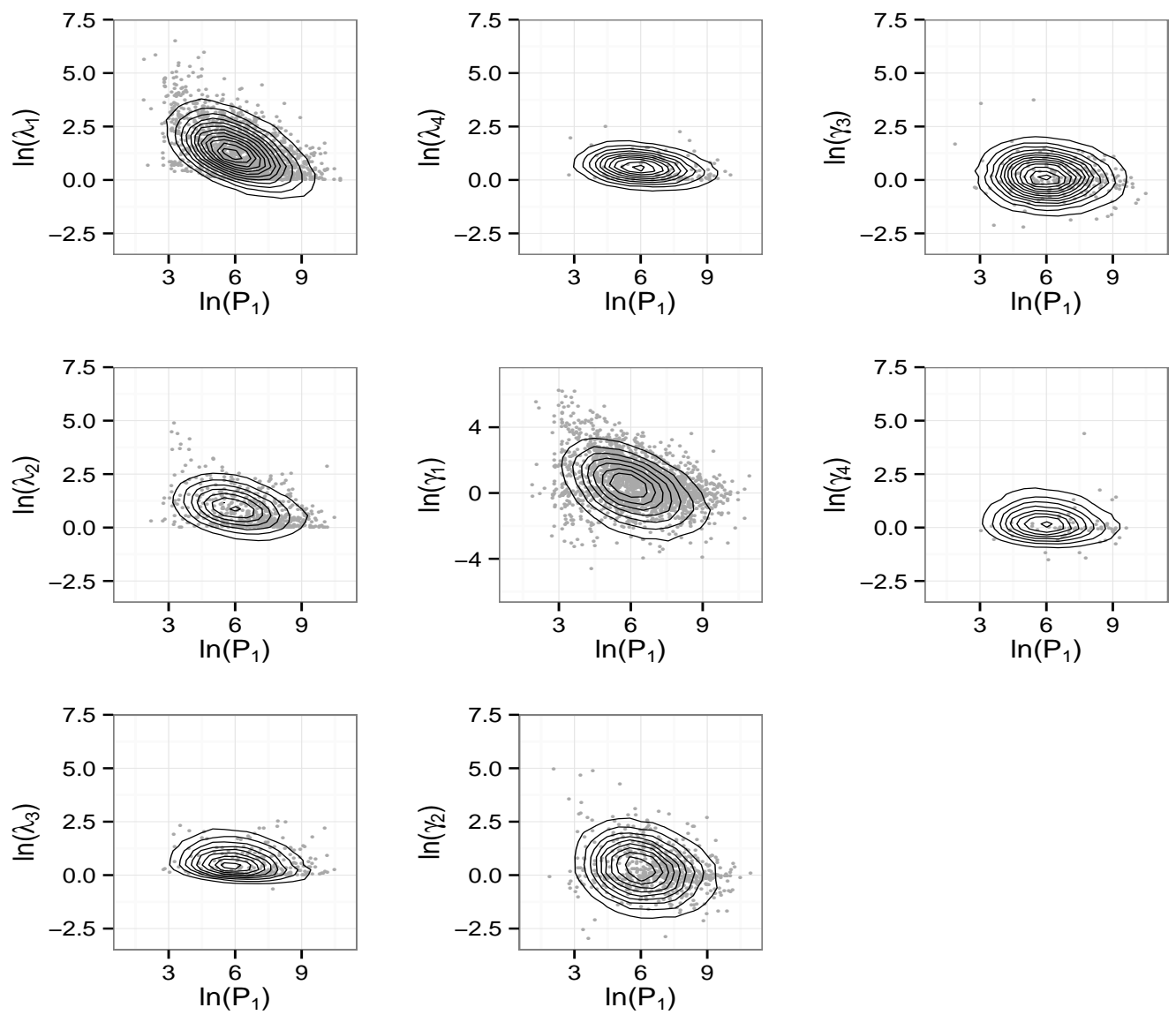

Figure 5: Empirical observations of the development vector $\boldsymbol{\Omega}$ (see (5p) and contour plots obtained from selected MSN model (see Table 5 . 
(which can be beyond the boundary of the triangle). Finally, we evaluate the total IBNR reserve. The prediction routine for the RBNP reserve is similar to the routine for IBNR claims. However, the number of RBNP claims is observed, and therefore does not require a simulation step.

RBNS reserves. For each RBNS claim $k$, we first simulate the remaining number of periods with payment. This implies simulating $U_{k}^{P}$ conditional on the (already) observed number of periods with payments (i.e. $t_{k}^{P}$ in Proposition 22. Analogously, we simulate the number of incurred loss adjustments, given the already observed number of periods with incurred loss adjustment $\left(t_{k}^{I}\right)$. Using these simulated values we generate the future part of the vector $\boldsymbol{\Omega}_{k}$ (see (8) and Proposition 22, given the observed part of this vector. Finally, we evaluate the RBNS reserve.

A comment applies to the simulation procedure for IBNR, RBNP and RBNS reserves. When the simulated $U_{k}^{P}$ or $U_{k}^{I}$ value exceeds the maximal dimension (4, respectively 4) used in the calibration process (in Section 5.2), we apply a tail factor ${ }^{1}$.

\begin{tabular}{rcccc}
\hline Approach & $\begin{array}{c}\text { Expected } \\
\text { Value }\end{array}$ & S.E. & VaR $_{0.95}$ & VaR $_{0.995}$ \\
\hline $\begin{array}{r}\text { Chain-Ladder } \\
\text { (payments only) }\end{array}$ & $15,261,478$ & $2,014,391$ & $18,787,651$ & $20,966,860$ \\
\hline $\begin{array}{r}\text { Chain-Ladder } \\
\text { (incurred losses only) }\end{array}$ & $20,337,149$ & $18,957,335$ & $27,474,358$ & $33,639,819$ \\
\hline PIC $\left(\rho_{1}=0\right)$ & $20,679,977$ & $1,918,866$ & $23,721,875$ & $25,677,209$ \\
\hline Wüthrich and Merz, 2010 & & & & \\
\hline $\begin{array}{r}\text { Individual paid-incurred } \\
\text { with MSN }\end{array}$ & $14,107,648$ & $1,684,473$ & $17,053,373$ & $19,872,889$ \\
\hline $\begin{array}{r}\text { Individual paid only } \\
\text { with MSN }\end{array}$ & $13,488,803$ & $1,840,184$ & $16,673,684$ & $19,883,007$ \\
\hline $\begin{array}{r}\text { Individual paid-incurred } \\
\text { with MN }\end{array}$ & $11,120,832$ & $1,194,498$ & $13,191,743$ & $14,850,789$ \\
\hline
\end{tabular}

Table 6: Outstanding loss reserves as estimated with techniques for aggregate data (chain-ladder, PIC with independence between paid and incurred loss development factors) and techniques for individual data (paid-incurred with MSN and MN specification and paid only with MSN specification). Expected values are from analytical expressions and other results are based on 10,000 simulations.

\subsubsection{Discussion}

Individual paid-incurred results. Table 6 summarizes the distribution of the outstanding loss reserve. Expected values are calculated with analytical expressions and other results are based on 10,000 simulations. As in Pigeon et al. (2013) estimates based on the MN specification are lower than the corresponding (and, preferred) MSN results. We also report estimates of the oustanding loss reserve obtained with a MSN model including information on payments only, using the structure $\boldsymbol{\Sigma}_{B}$ for the scale matrix. The inclusion of information from incurred amounts results in a larger expected value $(14,107,648$ versus $13,488,803)$ and in a smaller standard error $(1,684,473$ versus $1,840,184)$. The impact of these three individual reserving

\footnotetext{
${ }^{1}$ This tail factor is the geometric average of empirically observed development factors.
} 
models on the shape of the distribution of the total outstanding claim amount is illustrated in Figure 6

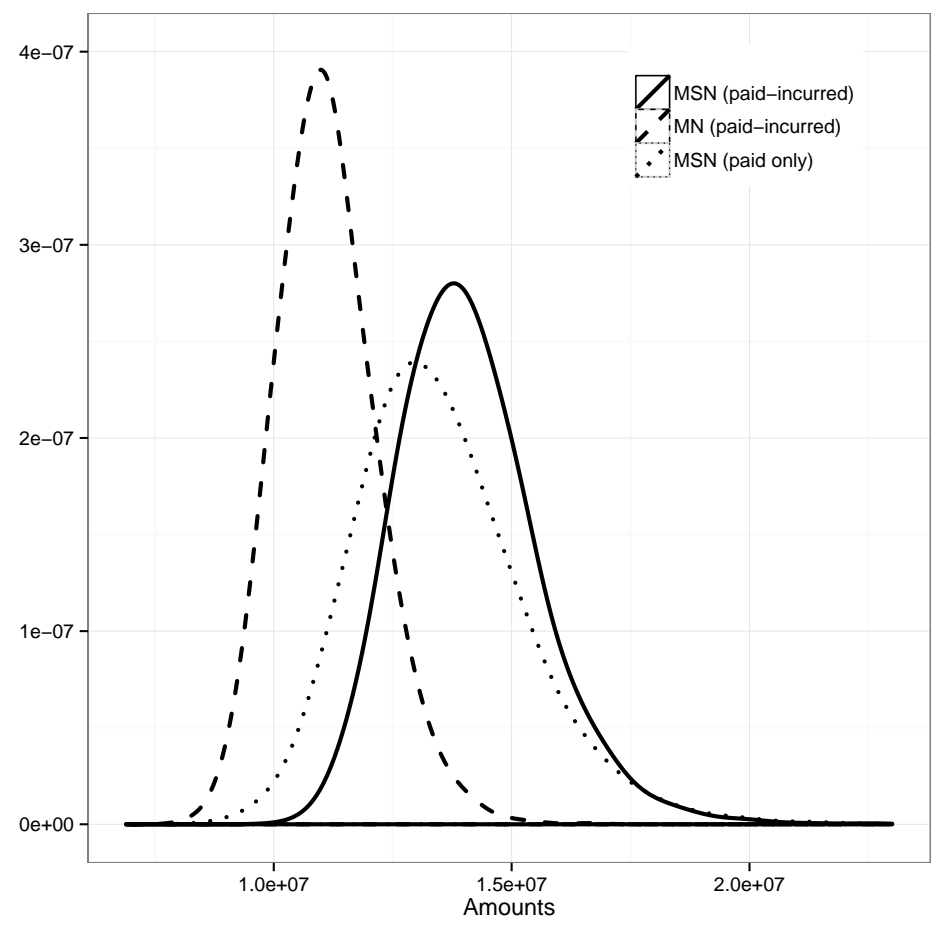

Figure 6: Probability density function of the total outstanding reserve obtained with the MSN model (solid line) and with the MN model (broken line) using information on paid and incurred amounts, and with the MSN model (dotted line) with information on paid amounts only. Results are based on 10,000 simulations.

Benchmarking with techniques for triangles. To enable benchmarking, we include the estimation results as obtained with standard techniques for run-off triangles. We include results for: a bootstrap Overdispersed Poisson (ODP) model with chain-ladder structure (as in the R chainladder package) on claim payments only (see Triangle 2 ) and on incurred losses only (see Triangle 3), and the PIC method with independence between paid and incurred loss development factors (Wüthrich and Merz (2010)). Despite the fact that the P/I ratio for the first occurrence period is $0.84<<1$, we also estimate the reserve with the Munich chain-ladder method (Quarg and Mack (2004)), as implemented in the $\mathrm{R}$ chainladder package. The corresponding best estimate is $22,293,102$ euro.

Benchmarking with claim experts' estimates. As reported in Section 5.1, the total, latest observed cumulative paid amount is 20,149,870 euro, while the most recent total incurred loss is $37,824,482$ euro. Thus, the claim experts' estimate of the total outstanding loss is $37,824,482-20,149,870=17,674,612$ euro. This is an aggregate, overall case estimate, as evaluated at the end of 2005, in order to cover future payments. A significant part of this amount (i.e. 1.18 million euro) comes from one unique claim. 
For claims occurred between the first day of 1997 and the last day of 2005, we also have information available on paid amounts and incurred losses as registered in 2006, 2007 and 2008. At the end of 2008, the total, latest observed cumulative paid amount is $28,251,441$ euro, while the total incurred loss amount is 36,745, 920 euro. We illustrate these results in Figure 7. Thus, 8, 101,571 euro was paid in 2006, 2007 and 2008, and the total case estimate is re-adjusted to $8,494,479$ euro (end of 2008). This results in a best estimate of 16,596,050 euro for the time horizon [2006, final run-off), using observed payments (in 2006, 2007 and 2008) and case estimates (set end of 2008). Taking into account the very large claim in the data set (with a case estimate of 988,871 euro, evaluated at the end of 2008), we conclude that the novel paidincurred reserving method for individual claims leads to reasonable results. For the case-study at hand, the techniques designed for triangles of paid and incurred data lead to remarkably higher estimates for the outstanding loss.

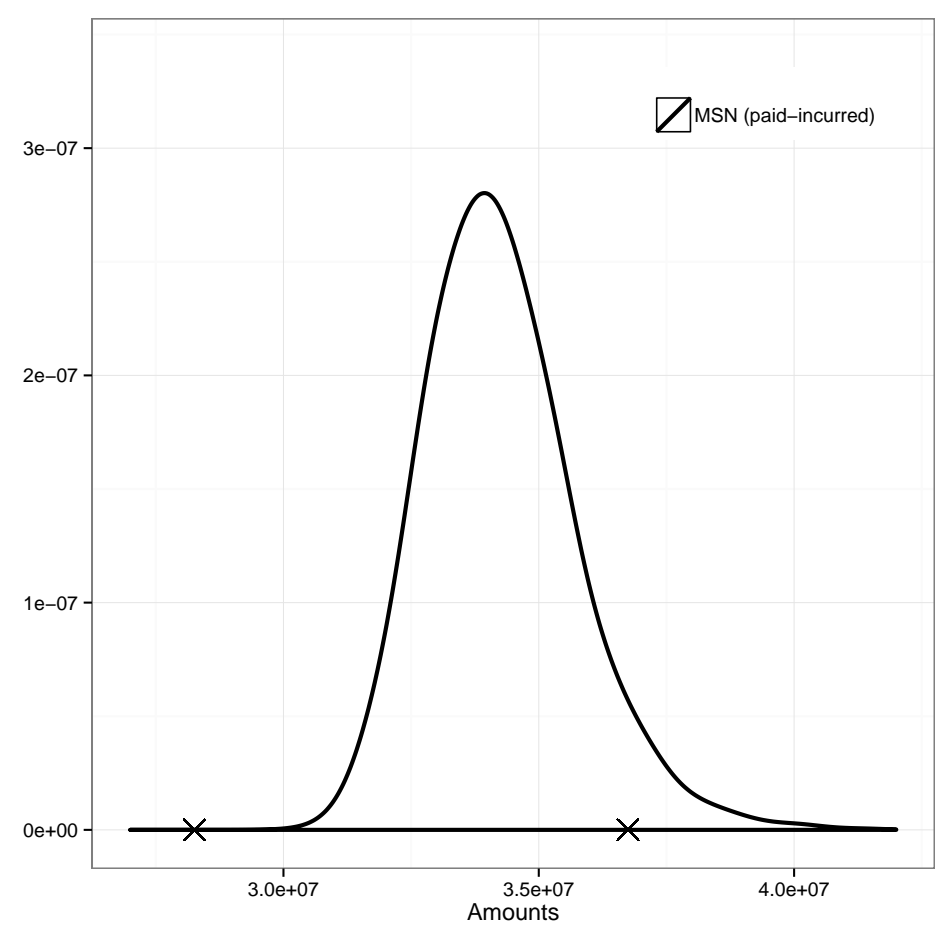

Figure 7: Probability density function of the total cumulative paid amount obtained with the MSN model, as evaluated end of 2005. Results are based on 10,000 simulations. Expected total cumulative paid amount is $20,149,870+14,088,172=34,238,042$ euro. The cross on the left along the horizontal axis $(28,251,441)$ represents the observed cumulative paid amount and the cross on the right along the horizontal axis $(36,745,920)$ represents the claim experts' estimate of the total cumulative paid amount, both evaluated at the end of 2008 .

\section{Conclusion}

In this paper, we have adopted the individual claim reserving model proposed by Pigeon et al. (2013) to combine information about paid amounts and incurred losses. The Multivariate Skew Normal distribution is used to describe the dynamics of the claim settlement process under the 
condition that the ultimate loss coincides for claim payments and incurred losses. Analytical expressions are derived for expected payments and incurred losses, as well as for their covariance structure, given the number of periods with claim payments and incurred loss adjustments. Conditional expected values, given past observed claim development, are also obtained in closed form so that the total outstanding IBNR and RBNP reserves, as well as the ultimate RBNS loss, are available analytically.

The theoretical results are illustrated by means of a case study based on a portfolio of general liability insurance policies. The Multivariate Skew Normal model is tested against its simpler Multivariate Normal counterpart and various covariances structures, supported by the data, are considered. AIC and BIC both support the Multivariate Skew Normal model developed in this paper, with an appropriate covariance matrix inducing dependence between the first payment and all development factors.

The comparison with collective reserving methods (performed in Table 6) reveals that - for the case study developed here - the proposed model in the present paper reduces the outstanding loss reserve and improves on predictive accuracy (lowering the associated standard errors). The same comment applies to the tails as VaRs at probability levels $95 \%$ and $99.5 \%$ also appear to be smaller with the Multivariate Skew Normal model compared to collective approaches. Considering the individual claim reserving model using paid data, only, as developed in Pigeon et al. (2013), we see that neglecting the information about incurred losses bias downwards the outstanding loss reserve but moderately impacts high quantiles. Replacing the Multivariate Skew Normal distribution with the Multivariate Normal one greatly underestimates both outstanding reserves and high quantiles. These numerical findings clearly support the practical relevance of the new individual claim reserving model developed in the present paper.

\section{References}

Akdemir, D. (2009). A class of Multivariate Skew Distributions: Properties and Inferential Issues. PhD thesis, Bowling Green State University, Ohio.

Akdemir, D. and Gupta, A. (2010). A matrix variate skew distribution. European Journal of Pure and Applied Mathematics, 3(2):128-140.

Antonio, K. and Plat, R. (2013). Micro-level stochastic loss reserving for general insurance. Scandinavian Actuarial Journal, in press.

Cheng, J. and Amin, N. (1983). Estimating parameters in continuous univariate distributions with a shifted origin. Journal of the Royal Statistical Society, Series B (Statistical Methodology), 45(3):394-403.

Drieskens, D., Henry, M., Walhin, J.-F., and Wielandts, J. (2012). Stochastic projection for large individual losses. Scandinavian Actuarial Journal, 2012(1):1-39.

Gupta, A. and Chen, J. (2004). A class of multivariate skew normal models. The Annals of the Institute of Statistical Mathematics, 56(2):305-315.

Haastrup, S. and Arjas, E. (1996). Claims reserving in continuous time: a nonparametric Bayesian approach. ASTIN Bulletin, 26(2):139-164.

Mack, T. (1993). Distribution-free calculation of the standard error of chain-ladder reserve estimates. ASTIN Bulletin, 23(2):213-225.

Norberg, R. (1993). Prediction of outstanding liabilities in non-life insurance. ASTIN Bulletin, 23(1):95-115. 
Norberg, R. (1999). Prediction of outstanding liabilities II. Model extensions variations and extensions. ASTIN Bulletin, 29(1):5-25.

Pigeon, M., Antonio, K., and Denuit, M. (2013). Individual loss reserving with the multivariate skew normal framework. ASTIN Bulletin, 43(3):399-428.

Posthuma, B., Veerkamp, E., and van Zwet, W. (2008). Combined analysis of paid and incurred losses. CAS E-forum Fall, pages 272-293.

Quarg, G. and Mack, T. (2004). Munich chain ladder. Blätter DGVFM, Band XXVI(597-630).

Ranneby, B. (1984). The maximum spacing method. An estimation method related to the maximum likelihood method. Scandinavian Journal of Statistics, 11(2):93-112.

Rosenlund, S. (2012). Bootstrapping individual claim histories. ASTIN Bulletin, 42(1):291-324.

Wüthrich, M. and Merz, M. (2010). Paid-incurred chain claims reserving method. Insurance: Mathematics and Economics, 46(3):568-579.

Wüthrich, M. V. and Merz, M. (2008). Stochastic Claims Reserving Methods in Insurance. Wiley Finance.

\section{A Moment generating function of $\mathrm{MSN}_{l}$ distribution}

The moment generating function (mgf) of a random vector $\mathbf{X} \sim \operatorname{MSN}_{l}\left(\boldsymbol{\mu}, \boldsymbol{\Sigma}^{1 / 2}, \boldsymbol{\Delta}\right.$ ) evaluated at $\mathbf{t} \in \mathbb{R}^{l}$ is

$$
m_{\mathbf{X}}(\mathbf{t})=2^{l} \cdot \exp \left(\mathbf{t}^{\prime} \boldsymbol{\mu}+0.5 \mathbf{t}^{\prime}\left(\boldsymbol{\Sigma}^{1 / 2}\right)\left(\boldsymbol{\Sigma}^{1 / 2}\right)^{\prime} \mathbf{t}\right) \cdot \prod_{j=1}^{l} \Phi\left(\frac{\Delta_{j}\left(\left(\boldsymbol{\Sigma}^{1 / 2}\right)^{\prime} \mathbf{t}\right)_{j}}{\sqrt{1+\Delta_{j}^{2}}}\right) .
$$

\section{B Proof of Proposition 1}

(i) By construction, $\boldsymbol{\Omega}_{v}$ follows a $\mathrm{MSN}_{u^{P+I}}$ distribution with parameters $\boldsymbol{\mu}_{v}, \boldsymbol{\Sigma}_{v}^{1 / 2}$ and $\boldsymbol{\Delta}_{v}$. Let $\ln \left(P_{t}\right)=\ln \left(P_{1}\right)+\sum_{j=1}^{t-1} \ln \left(\lambda_{j}\right), t=2, \ldots, u^{P}+1$ and $\ln \left(I_{t}\right)=\ln \left(P_{1}\right)+\sum_{j=1}^{u^{P}} \ln \left(\lambda_{j}\right)+$ $\sum_{j=t}^{u^{I}} \ln \left(\gamma_{j}\right), t=1, \ldots, u^{I}$. The moment generating function of the random vector $\mathbf{D}=$ $\mathbf{R} \boldsymbol{\Omega}_{v}$ is specified as follows (with $\mathbf{t}$ a $u^{P+I}$ column vector)

$$
\begin{aligned}
& m_{\mathbf{D}}(\mathbf{t})=m_{\boldsymbol{\Omega}_{v}}\left(\mathbf{R}^{\prime} \mathbf{t}\right) \\
& =2^{u^{P+I}} \cdot \exp \left(\mathbf{t}^{\prime} \mathbf{R} \boldsymbol{\mu}_{v}+0.5 \mathbf{t}^{\prime} \mathbf{R}\left(\boldsymbol{\Sigma}_{v}^{1 / 2}\right)\left(\boldsymbol{\Sigma}_{v}^{1 / 2}\right)^{\prime} \mathbf{R}^{\prime} \mathbf{t}\right) \cdot \prod_{j=1}^{u^{P+I}} \Phi\left(\frac{\Delta_{v, j}\left(\left(\boldsymbol{\Sigma}_{v}^{1 / 2}\right)^{\prime} \mathbf{R}^{\prime} \mathbf{t}\right)_{j}}{\sqrt{1+\Delta_{v, j}^{2}}}\right) .
\end{aligned}
$$

We recognize the moment generating function of a MSN distribution and conclude

$$
\mathbf{D} \sim \operatorname{MSN}_{u^{P+I}}\left(\mathbf{R} \boldsymbol{\mu}_{v}, \mathbf{R} \boldsymbol{\Sigma}_{v}^{1 / 2}, \boldsymbol{\Delta}_{v}\right) .
$$

(ii) For $t=1, \ldots, u^{P}+1$, we have, conditional on $U^{P}$ and $U^{I}, E\left[P_{t}\right]=E\left[e^{\ln \left(P_{t}\right)}\right]=E\left[e^{\mathbf{R}_{[t,]} \boldsymbol{\Omega}_{v}}\right]=$ $m_{\boldsymbol{\Omega}_{v}}\left(\mathbf{R}_{[t,]}^{\prime}\right)$, so 16$)$ allows us to write

$$
E\left[P_{t}\right]=2^{u^{P+I}} \cdot \exp \left(\mathbf{R}_{[t,]} \boldsymbol{\mu}_{v}+0.5 \mathbf{R}_{[t,]}\left(\boldsymbol{\Sigma}_{v}^{1 / 2}\right)\left(\boldsymbol{\Sigma}_{v}^{1 / 2}\right)^{\prime} \mathbf{R}_{[t,]}^{\prime}\right) \cdot \prod_{j=1}^{u^{P+I}} \Phi\left(\frac{\Delta_{v, j}\left(\left(\boldsymbol{\Sigma}_{v}^{1 / 2}\right)^{\prime} \mathbf{R}_{[t,]}^{\prime}\right)_{j}}{\sqrt{1+\Delta_{v, j}^{2}}}\right),
$$


with $\mathbf{R}_{[t,]}$ the $t^{t h}$ row of $\mathbf{R}$. The proof is similar for $E\left[I_{t}\right], t=1, \ldots, u^{I}$.

(iii) For $i, j=1, \ldots, u^{P+I}$, we have, conditional on $U^{P}$ and $U^{I}$,

$$
\begin{aligned}
\operatorname{Cov}\left[e^{D_{i}}, e^{D_{j}}\right] & =E\left[e^{D_{i}+D_{j}}\right]-E\left[e^{D_{i}}\right] E\left[e^{D_{j}}\right] \\
& =E\left[e^{\mathbf{t}_{i+j} \mathbf{R} \boldsymbol{\Omega}_{v}}\right]-E\left[e^{\mathbf{R}_{[i,]} \boldsymbol{\Omega}_{v}}\right] E\left[e^{\mathbf{R}_{[j,]} \boldsymbol{\Omega}_{v}}\right] \\
& =m_{\mathbf{R} \boldsymbol{\Omega}_{v}}\left(\mathbf{t}_{i+j}^{\prime}\right)-m_{\boldsymbol{\Omega}_{v}}\left(\mathbf{R}_{[i,]}^{\prime}\right) m_{\boldsymbol{\Omega}_{v}}\left(\mathbf{R}_{[j,]}^{\prime}\right) .
\end{aligned}
$$

By using results in $(i)$ and $(i i)$, expression $(12)$ follows.

\section{Proof of Proposition 2}

We define

$$
\boldsymbol{\Psi}:=\left[\begin{array}{cc}
\mathbf{1}_{|v o| \times|v o|} & \mathbf{0}_{|v o| \times|v f|} \\
-\tilde{\mathbf{\Sigma}}_{f o} \tilde{\boldsymbol{\Sigma}}_{o o}^{-1} & \mathbf{1}_{|v f| \times|v f|}
\end{array}\right] .
$$

Using a similar argument as in $(i)$ of Proposition 1, the random vector

$$
\boldsymbol{\Psi} \boldsymbol{\Omega}_{v^{\prime}}=\left[\begin{array}{ll}
\boldsymbol{\Omega}_{v o} & \boldsymbol{\Omega}_{v f}^{\star}=\boldsymbol{\Omega}_{v f}-\tilde{\boldsymbol{\Sigma}}_{f o} \tilde{\boldsymbol{\Sigma}}_{o o}^{-1} \boldsymbol{\Omega}_{v o}
\end{array}\right]^{\prime},
$$

follows a $\mathrm{MSN}_{u^{P+I}}$ distribution with parameters

$$
\boldsymbol{\Psi} \boldsymbol{\mu}_{v^{\prime}}=\left[\begin{array}{c}
\tilde{\boldsymbol{\mu}}_{o} \\
\tilde{\boldsymbol{\mu}}_{f}-\tilde{\boldsymbol{\Sigma}}_{f o} \tilde{\boldsymbol{\Sigma}}_{o o}^{-1} \tilde{\boldsymbol{\mu}}_{o}
\end{array}\right], \quad \boldsymbol{\Psi} \boldsymbol{\Sigma}_{v^{\prime}}^{1 / 2}=\left[\begin{array}{cc}
\tilde{\boldsymbol{\Sigma}}_{o o} & \mathbf{0} \\
\mathbf{0} & \tilde{\boldsymbol{\Sigma}}_{f f}
\end{array}\right], \quad \boldsymbol{\Delta}_{v^{\prime}}=\left[\begin{array}{c}
\tilde{\boldsymbol{\Delta}}_{o} \\
\tilde{\boldsymbol{\Delta}}_{f}
\end{array}\right] .
$$

The probability density function of $\boldsymbol{\Psi} \boldsymbol{\Omega}_{v^{\prime}}$ becomes

$$
\begin{gathered}
\frac{2^{|v o|}}{\operatorname{det}\left(\tilde{\boldsymbol{\Sigma}}_{o o}\right)^{1 / 2}} \cdot \phi_{|v o|}\left(\tilde{\boldsymbol{\Sigma}}_{o o}^{-1 / 2}\left(\boldsymbol{\omega}_{v o}-\tilde{\boldsymbol{\mu}}_{o}\right)\right) \cdot \prod_{j=1}^{|v o|} \Phi\left(\Delta_{v^{\prime}, j} \mathbf{e}_{j}^{\prime} \tilde{\boldsymbol{\Sigma}}_{o o}^{-1 / 2}\left(\boldsymbol{\omega}_{v o}-\boldsymbol{\mu}_{o}\right)\right) \\
\cdot \frac{2^{|v f|}}{\operatorname{det}\left(\tilde{\boldsymbol{\Sigma}}_{f f}\right)^{1 / 2}} \cdot \phi_{|v f|}\left(\tilde{\boldsymbol{\Sigma}}_{f f}^{-1 / 2}\left(\boldsymbol{\omega}_{v f}^{\star}-\left(\tilde{\boldsymbol{\mu}}_{f}-\tilde{\boldsymbol{\Sigma}}_{f o} \tilde{\boldsymbol{\Sigma}}_{o o}^{-1} \tilde{\boldsymbol{\mu}}_{o}\right)\right)\right) \\
\cdot \prod_{j=|v o|+1}^{|v|} \Phi\left(\Delta_{v^{\prime}, j} \mathbf{e}_{j}^{\prime} \tilde{\boldsymbol{\Sigma}}_{f f}^{-1 / 2}\left(\boldsymbol{\omega}_{v f}^{\star}-\left(\tilde{\boldsymbol{\mu}}_{f}-\tilde{\boldsymbol{\Sigma}}_{f o} \tilde{\boldsymbol{\Sigma}}_{o o}^{-1} \tilde{\boldsymbol{\mu}}_{o}\right)\right)\right) .
\end{gathered}
$$

We recognize a product of two MSN densities

$$
\operatorname{MSN}_{|v o|}\left(\tilde{\boldsymbol{\mu}}_{o}, \tilde{\boldsymbol{\Sigma}}_{o o}^{1 / 2}, \tilde{\boldsymbol{\Delta}}_{o}\right) \times \operatorname{MSN}_{|v f|}\left(\tilde{\boldsymbol{\mu}}_{f}-\tilde{\boldsymbol{\Sigma}}_{f o} \tilde{\boldsymbol{\Sigma}}_{o o}^{-1} \tilde{\boldsymbol{\mu}}_{o}, \tilde{\boldsymbol{\Sigma}}_{f f}^{1 / 2}, \tilde{\boldsymbol{\Delta}}_{f}\right) .
$$

Therefore, the components $\boldsymbol{\Omega}_{v o}$ and $\boldsymbol{\Omega}_{v f}-\tilde{\boldsymbol{\Sigma}}_{f o} \tilde{\boldsymbol{\Sigma}}_{o o}^{-1} \boldsymbol{\Omega}_{v o}$ are independent. By substituting $\boldsymbol{\omega}_{v f}^{\star}=$ $\boldsymbol{\omega}_{v f}-\tilde{\boldsymbol{\Sigma}}_{f o} \tilde{\boldsymbol{\Sigma}}_{o o}^{-1} \boldsymbol{\omega}_{v o}$ in 17 , we find that given $\boldsymbol{\Omega}_{v o}=\boldsymbol{\omega}_{v o}, \boldsymbol{\Omega}_{v f}$ is MSN distributed with parameters $\tilde{\boldsymbol{\mu}}_{f}+\tilde{\boldsymbol{\Sigma}}_{f o} \tilde{\boldsymbol{\Sigma}}_{o o}^{-1}\left(\boldsymbol{\omega}_{v o}-\tilde{\boldsymbol{\mu}}_{o}\right), \tilde{\boldsymbol{\Sigma}}_{f f}$ and $\boldsymbol{\Delta}_{f}$. Finally, using the approach in the proof of Proposition 1 (ii) (see Appendix B), the expression for

$$
E\left[P_{t^{P}+s} \mid \boldsymbol{\Omega}_{v o}=\boldsymbol{\omega}_{v o}, U^{P}=u^{P}, U^{I}=u^{I}\right] .
$$

follows. 
FACULTY OF ECONOMICS AND BUSINESS Naamsestraat 69 bus 3500

3000 LEUVEN, BELGIË

tel. + 3216326612

fax +3216326791

info@econ.kuleuven.be www.econ.kuleuven.be 